\title{
Estimativas de mortalidade para 0 Rio Grande do Norte em um contexto de pré-transição demográfica
}

\author{
Luciana Conceição de Lima* \\ Dayane Julia Carvalho Dias ${ }^{\star \star}$ \\ Luana Junqueira Dias Myrrha ${ }^{\star \star \star}$
}

\begin{abstract}
Para períodos anteriores ao início da transição demográfica há poucos trabalhos quantitativos sobre o impacto da mortalidade na população. Com base nessa lacuna, o presente artigo estimou indicadores de mortalidade para o Rio Grande do Norte no contexto de pré-transição demográfica, utilizando dados do século XIX provenientes de mapas estatísticos de população (1801 e 1805) e de recenseamentos (1872 e 1890). Para lidar com as informações de população e óbito, empregou-se método de Growth Balance de Brass (1975), tabelas de vida de Coale e Demeny (1996), funções extraídas do The Human Mortality Database e técnica de padronização indireta. Como resultados, verificou-se que a mortalidade no Rio Grande do Norte se mostrou mais intensa na população infantil e naquela acima de 50 anos, que representam os segmentos mais vulneráveis às condições adversas como as secas e as doenças infecciosas. Obteve-se também uma expectativa de vida ao nascer de 32,6 anos, valor muito próximo às estimativas de Mortara (1941) para o Brasil entre 1870 e 1890. Sugerem-se novos estudos para o período de pré-transição demográfica e maior utilização de técnicas quantitativas em análises voltadas para o passado brasileiro.
\end{abstract}

Palavras-chave: Pré-transição demográfica. Mortalidade. Rio Grande do Norte. Técnicas demográficas.

\footnotetext{
*Universidade Federal do Rio Grande do Norte (UFRN), Natal-RN, Brasil (limamarx@gmail.com).

** Universidade Estadual de Campinas, Núcleo de Estudos de População “Elza Berquó” (Unicamp/Nepo), Campinas-SP, Brasil (dayanejuliacd@gmail.com).

${ }^{\star \star \star}$ Universidade Federal do Rio Grande do Norte (UFRN), Natal-RN, Brasil (luanamyrrha@gmail.com).
} 


\section{Introdução}

No Brasil, a transição da mortalidade somente ocorreu por volta da década de 1940, quando políticas explícitas de saúde pública e de saneamento básico contribuíram decisivamente para a redução do volume de óbitos na população (PRATA, 1992; WOOD; CARVALHO, 1998; ALVES, 2002). Para períodos históricos anteriores a essa transição há escassez de estatísticas que dimensionem o impacto da mortalidade na população. Segundo Marcílio (1973), antes da era estatística no Brasil (cujos marcos são o primeiro Recenseamento Geral de 1872 e a oficialização do registro das estatísticas civis em 1916), eram poucas as estimativas populacionais gerais e, embora houvesse relativa abundância na disponibilidade de fontes, como os registros paroquiais, a qualidade e a abrangência dessas informações não eram as mesmas para todas as localidades.

No caso dos mapas estatísticos de população, que se tornaram obrigatórios em todos os territórios sob o domínio do Império português a partir de 1776 (ALDEN, 1963), a qualidade dos primeiros levantamentos era limitada devido aos desafios nos séculos iniciais da colonização em "conhecer” essa população (PAIVA et al., 2012). Problemas como o sub-registro das informações eram frequentes, além do fato de, em um território de dimensões continentais e em face dos recursos disponíveis na época para a coleta dos dados, serem consideráveis as dificuldades em realizar levantamentos demográficos nas áreas mais remotas (BOTELHO, 1998).

Essas dificuldades metodológicas associadas às fontes de informações disponíveis para os períodos pré-estatístico e proto-estatístico dificultam uma análise empírica acerca do comportamento das variáveis demográficas no período de pré-transição. Segundo Nadalin (2004), embora níveis elevados de mortalidade sejam emblemáticos do passado brasileiro (e em virtude, sobretudo, da eclosão de doenças infecciosas e práticas precárias de higiene), possivelmente, houve mais de um sistema demográfico no período anterior à transição demográfica e com importantes variações regionais.

Levando-se em conta esses aspectos, o objetivo do presente estudo é estimar indicadores de mortalidade para o Rio Grande do Norte no contexto de pré-transição demográfica. Tendo em vista que até aproximadamente 1940 os indicadores de mortalidade permaneceram praticamente inalterados, para estimá-los (basicamente, funções de mortalidade e a esperança de vida ao nascer) para o Rio Grande do Norte em uma fase anterior ao declínio dos níveis de mortalidade, utilizaram-se mapas estatísticos de população e de óbitos referentes ao século XIX: mapas de 1801 e de 1805, disponibilizados pelo projeto Counting Colonial Populations: demography and the use of statistics in the Portuguese Empire, 1776-1890 da Universidade Nova de Lisboa. Ademais, foram incluídas informações do Recenseamento Geral do Império de 1872 e de 1890 para estimativas de população.

Para obter a função de mortalidade e a esperança de vida ao nascer, incialmente foi necessário corrigir o denominador das taxas de mortalidade, ou seja, a população do Rio 
Grande do Norte, em 1805, por idade, utilizando a retroprojeção da população recenseada em 1872. Para estimar o número de óbitos, aplicaram-se técnicas indiretas de mortalidade, tendo em vista a já conhecida fragilidade dos dados históricos em termos, principalmente, de sub-registro das informações de população e óbitos. Para correção da mortalidade, empregou-se o método de Growth Balance de Brass (1975) e, para estimação das funções de mortalidade e obtenção da esperança de vida ao nascer, foram utilizadas as tabelas de vida de Coale e Demeny (1996), funções extraídas do The Human Mortality Database e, também, técnica de padronização indireta para compatibilização das funções de mortalidade infanto-juvenil e adulta estimadas.

Esse artigo se subdivide em quatro seções. A seguir, apresenta-se um breve panorama sobre aspectos históricos e econômicos do Rio Grande do Norte e, também, sobre a mortalidade na capitania e depois província potiguar no final do século XVIII e início do XIX. Posteriormente, é descrita a metodologia utilizada (materiais e métodos) e discutem-se os principais resultados encontrados. Por fim, são apresentadas as conclusões desse estudo.

\section{Da conquista da capitania no século XVI ao final do século XIX: aspectos históricos e econômicos do Rio Grande do Norte}

A capitania do Rio Grande do Norte foi definitivamente conquistada em 1597, quando o então governador geral do Brasil D. Francisco de Souza decidiu expulsar os franceses, conforme Cartas Régias de 9 de novembro de 1596 e 15 de março de 1597 (SANTOS, 1994; LYRA, 2008). No ano seguinte à conquista da capitania, foi construída a Fortaleza dos Santos Reis Magos, em referência ao dia seis de janeiro consagrado aos Santos Reis, data em que foi iniciada a edificação do forte. Esse foi o marco da posse portuguesa das terras potiguares e, também, estratégia dos colonizadores para defendê-la contra invasores (MEDEIROS, 1973; SANTOS, 1994).

Alguns anos mais tarde, em 25 de dezembro de 1599, fundou-se nas proximidades do forte a cidade de Natal. Do século XVI até início do XIX a capital potiguar era apenas uma povoação sem destaque, assim como toda a capitania do Rio Grande do Norte. Segundo Lyra (1972, p. 27):

Conquistado desde o fim do século XVI, o Rio Grande do Norte era ainda, no começo do século XIX, uma das capitanias mais pobres e atrasadas do Brasil; e a sua capital, que fora fundada em 1599, não passava de uma povoação sem importância, que, medindo, em 1757 , cerca de quatrocentas braças de comprimento por cinquenta de largura e contendo cento e dezoito casas, tinha, em 1817, apenas 700 habitantes.

O processo de ocupação do território da capitania do Rio Grande do Norte para além da faixa litorânea foi interrompido pela ocupação holandesa entre 1633 e 1654. Estabelecendo alianças com os indígenas, que, por sua vez, queriam permanecer em suas terras, os holandeses exploraram não somente a costa da capitania, mas também a região do agreste (SANTOS, 1994; DIAS, 2015). 
De um modo geral, os historiadores potiguares asseguram que a região mais povoada e utilizada pelo holandês compreendia o agreste, Natal até Canguaretama, próximo dos rios torrenciais. Os produtos eram embarcados para Recife, e Natal, e na embocadura dos rios Pirangi, Camurupim e Barra do Cunhaú. 0 sertão não foi explorado pelo holandês. (SANTOS, 1994, p.41-42)

Restaurada a paz com o fim da ocupação holandesa, o movimento colonizador se reiniciava, porém, ainda restrito ao litoral e entre as ribeiras do Potengi, Cunhaú e CearáMirim, que eram áreas mais favoráveis à lavoura (LYRA, 2008). 0 povoamento efetivo do interior da capitania veio somente no século XVIII, sobretudo com a expansão da criação de gado, uma vez que os criadores buscavam as ricas pastagens do sertão para desenvolvimento dessa atividade (MEDEIROS, 1973). São exemplos de estruturas de poder instaladas para controle e gerenciamento de colonos da pecuária o Arraial do Queiquó (1700), o Regimento de Ordenanças da Ribeira do Seridó (1726), a Povoação do Caicó (1735), a Freguesia da Gloriosa Senhora Santa Ana do Seridó (1748) e a Vila Nova do Príncipe (1788) (MACEDO, 2011).

O sertão voltava-se para a pecuária e o litoral para a atividade açucareira. Data de 1614 o início da produção do engenho de Cunhaú, que era o principal da capitania (SANTOS, 1994; DIAS, 2015). Somente no final do século XVIII são encontradas referências sobre outros engenhos no Rio Grande, instalados em Natal e na Vila de São José, ou seja, há menção sobre unidades produtoras de açúcar apenas no litoral potiguar (DIAS, 2011).

Na segunda metade do século XVIII, a capitania do Rio Grande do Norte enfrentou importantes períodos de seca, como as de 1754, 1760 e 1772. Além de representarem a destruição de lavouras e morte do gado, as grandes secas amplificaram ainda mais o conflito por terras entre colonizadores e índios (SANTOS, 1994). Em virtude desses conflitos, das epidemias de varíola e das próprias secas, o contingente indígena na população da capitania reduziu-se de maneira importante. Por outro lado, crescia a demanda por mão de obra escrava negra ainda no século XVIII, sobretudo para a exploração da cana-de-açúcar (MEDEIROS, 1973). Aliás, o redirecionamento do foco da produção econômica do sertão para a atividade açucareira e, mais tarde, para a produção de algodão e exploração do sal constitui fator marcantes do século XIX no Rio Grande do Norte:

[...] a tradicional agricultura versus pecuária, na Província do Rio Grande do Norte, vai ser substituída pela indústria açucareira, de modo como informava o presidente em exercício no ano de 1853 - "é nela que a Província começa a depositar todas as suas esperanças" [...]. No segundo quartel do século, os estabelecimentos salineiros elevaram-se a um nível de boa escala industrial, principalmente os situados em Açu, Mossoró, Macau e Areia Branca. Paralelamente, nas áreas mais afetadas pelas secas, intensificou-se o cultivo do algodoeiro, a ele se dedicando muito matuto, que até então aplicava no pastoreio toda a sua atividade. (MEDEIROS, 1973, p. 68-69)

Foi no século XIX que a capitania e posteriormente província do Rio Grande do Norte conheceu sua maior seca. Em 1877, a situação de calamidade associada à eclosão de 
epidemias como a varíola impulsionou um grande contingente das áreas mais afetadas do sertão para o litoral e agreste como Areia Branca, Mossoró e Macau (SANTOS, 1994). Segundo Medeiros (1973), a tragédia das secas influenciou decisivamente o baixo desenvolvimento econômico da província potiguar, em comparação com as demais, tendo em vista a ausência de corrente migratória de outros povos para suprir as perdas populacionais do período. No próximo item, é discutida essa sinergia entre secas e epidemias que, possivelmente, impactaram a estrutura da população potiguar no período analisado.

\section{Secas e epidemias: a mortalidade no Rio Grande do Norte em um contexto de pré-transição demográfica}

Segundo Monteiro (2002), importantes transformações envolveram o Rio Grande do Norte entre o final do século XVIII e as primeiras décadas do século XIX: substituição do monopólio comercial português pela liberdade comercial destacada pela presença da economia inglesa; passagem da condição de colônia para nação politicamente independente; e necessidade de reorganização política, com vistas a manter o monopólio dos grandes proprietários rurais e, ao mesmo tempo, garantir a unidade territorial.

Todavia, em meio a essas importantes mudanças, a sucessão de secas assolava as capitanias do norte. Entre 1791 e 1793 ocorreu a maior seca do século XVIII, atingindo não somente a capitania do Rio Grande do Norte, mas também Ceará, Pernambuco, Bahia, Sergipe, Alagoas, Paraíba e Piauí. Além das pestes de gafanhotos, cobras e ratos, as secas vinham acompanhadas de epidemias como a de varíola (popularmente conhecida como bexiga), produzindo ainda mais mortos (GUERRA, 1981). Segundo padre Vieira, sobre a seca da ribeira do Apody durante 1792 e 1793 no Rio Grande do Norte:

0 grande desamparo em que a Providencia e a natureza os entregaram ao jogo dos tempos os encheu de receios e de temores tantos que se vira obrigados por tudo a procurar, ávidos da conservação da cara vida, que é preciosa e estimavel ao homem [...]. De sorte que os agreste e desconhecidos alimentos, por suas qualidades, delecterios da saude e da vida daqueles habitadores produziram nelles inchações disformes, vomitos de sangue extraordinários, dysenterias ferinas, males cutâneos crueis, marasmos ultimos; vindo por esse motivo a povoar as sepulturas dos campos e dos povoados. (LYRA, 1981, p. 53-54)

Tanto no período colonial quanto no tempo do Império, as medidas governamentais para tentar resolver o problema das secas eram pouco efetivas e preventivas, ocasionando episódios sucessivos de calamidades públicas (GUERRA, 1981). Entre 1877 e 1879, a região conheceu seu maior período de seca do século XIX.

Sobre a situação em Mossoró, pequena cidade do Rio Grande do Norte que foi o ponto convergente dos retirantes do interior na seca de 1877 a 1879, Felipe Guerra descreve (1981, p. 213-214):

Nenhum abrigo, nenhum abarracamento. Os retirantes espalhavam-se pelos campos, pelas ruas, pelos arredores da cidade. Valados eram abertos no cemitério. Pessoas 
pagas recolhiam ao valado cadáveres encontrados pelas calçadas, pelas praças, por toda a parte. Depois de cheio, as pás e enxadas cobriam de terra os restos das infelizes vítimas da desgraça.

Embora na segunda metade do século XIX o Rio Grande do Norte estivesse em uma fase de "prosperidade econômica", o estado de salubridade da província era precário, com condições sanitárias irregulares que facilitavam a propagação de doenças infecciosas (ARAÚJO; MACEDO, 2011), tais como cólera-morbo, febre amarela e varíola (SANTOS, 2013). A varíola, presente entre a população brasileira desde o período colonial, fez muitas vítimas no Rio Grande do Norte durante quase todo o século XIX. Consta registro da febre amarela na capitania em setembro de 1850 (em Natal, Assú e outras localidades do interior) (FRANCO, 1969) e a cólera-morbo, segundo Cascudo (1984), surgiu no Rio Grande do Norte em 1856 , provocando a morte de 2.563 pessoas.

No próximo item apresentam-se os materiais e métodos utilizados para o alcance do objetivo proposto de estimar indicadores de mortalidade para o Rio Grande do Norte no contexto da pré-transição demográfica, utilizando fontes de dados disponíveis para o século XIX.

\section{Materiais e métodos}

As fontes de dados utilizados foram os mapas estatísticos de população (dados de população e óbito) e informações dos Recenseamentos de $1872^{1}$ e de $1890^{2}$ (dados de população). Os mapas estatísticos referentes a 1801 e 1805 se encontravam depositados no Arquivo Histórico Ultramarino de Lisboa (AHU) e disponibilizados para fins desse artigo pelo projeto Counting Colonial Populations: demography and the use of statistics in the Portuguese Empire, 1776-1890 da Universidade Nova de Lisboa.

Mapas estatísticos de população de 1801 e 1805

Os Estados sempre tiveram necessidade de enumerar, mensurar e quantificar suas populações, riquezas e recursos com o objetivo de conhecer a nação por meio da estatística. De início, essa demanda ocorreu devido ao recrutamento de soldados para as guerras e depois para legislar e administrar inúmeros aspectos das esferas públicas e privadas. Com o desenvolvimento do mundo moderno e do capitalismo, emergiram as difíceis questões da natalidade, mortalidade, longevidade, saúde pública, habitação, migração, entre outras (SENRA, 2006).

O primeiro levantamento de caráter censitário do Brasil ocorreu em 1776 e constituiu um marco de mudança para o período proto-estatístico (BOTELHO, 1998). Esse levantamento era realizado por meio de mapas de população na gestão do marquês de Pombal, que trouxe

\footnotetext{
$\overline{1}$ Disponivel em: 〈http://www.nphed.cedeplar.ufmg.br/pop72/〉.

${ }^{2}$ Disponível em: 〈http://biblioteca.ibge.gov.br/visualizacao/livros/liv49677.pdf〉.
} 
um caráter modernizante inspirado nas ideias europeias de levantamento populacional (PAIVA et al., 2012), com o objetivo de "conhecer a realidade brasileira" para promover o renascimento agrícola da colônia e produção de matérias-primas para a industrialização de Portugal (BOTELHO, 1998).

Segundo Paiva (2015), os mapas que apresentam maior grau de detalhamento de suas informações são os decorrentes dos diplomas régios de 1796 e 1797, tais como os mapas de 1801 e 1805 . Com base nas características dos mapas utilizados para a capitania do Rio Grande do Norte em 1801 e 1805, observa-se que nestes documentos constavam informações da população por grupos de idade (quinquenais e decenais) e sexo, segundo categorias de cor (branco, preto e mulato), estado civil (casado, solteiro e viúvo) e condição (livre, escravo ou indígena), sendo os indígenas aqueles "domesticados” e não os que de fato habitavam a capitania. Também constam informações de nascimento, casamento e óbito. Os dados de casamentos se encontram desagregados por grupos etários decenais (concentrados entre 10 e 70 anos), sexo, cor (branco, negro e pardo) e condição (livre e cativo). Os nascimentos estão registrados por sexo, cor (branco, negro e pardo), condição (livre e cativo) e nascidos vivos, mortos e gêmeos. Para os óbitos, as informações aparecem por grupos de idade (quinquenais até dez anos e decenais até 100 anos), sexo, cor (branco, negro e pardo) e condição (livre e cativo). Além disso, há dados sobre ocupações dos habitantes.

Apesar da confecção de mapas estatísticos, o simples trabalho não era sinônimo de qualidade. Pouco se seguia o modelo padrão imposto pela administração provincial para a coleta de dados e os responsáveis por ela nem sempre eram suficintemente qualificados para tal função. Não havia rigor estatístico e nem sempre os dados englobavam toda a província, redundando na baixa qualidade da informação (MARTINS; LIMA; SILVA, 2002).

Portanto, supunha-se que durante a Colônia e o Império brasileiro uma parcela considerável dos óbitos e dos nascimentos não era contabilizada. A obrigatoriedade de registro civil de nascimentos, casamentos e óbitos ocorreu apenas em 1888 (FAGGION, 2000). Além disso, faltavam equipe técnica qualificada e recursos financeiros para recensear toda a população em um país de dimensões continentais (BASSANEZI; BACELLAR, 2002). No entanto, mesmo com a existência de todas as dificuldades para a realização de um censo nacional, pouco a pouco houve a consolidação da ordenação dos registros administrativos e, com isso, possibilitou-se a organização de estatísticas demandadas.

Para avaliar a qualidade das informações foram empregadas técnicas demográficas de correção de população e óbito. Esses procedimentos metodológicos são descritos no próximo item.

\section{Correção dos dados de população}

Estimou-se a população de 1805 com base naquela recenseada em 1872, considerando a distribuição proporcional por grupo etário da população recenseada em 1890 . 
Em análise preliminar, verificou-se que a estrutura etária da população do Rio Grande do Norte registrada pelo Recenseamento de 1872 era mais envelhecida em relação àquela de 1890, o que em princípio representa uma contradição. Assim, com base na distribuição proporcional da população por grupo etário do Recenseamento de 1890 , recalculou-se a distribuição proporcional da população por grupo etário do Recenseamento de 1872. Dessa forma, supondo-se constante o crescimento da população por grupo etário no período de pré-transição, de 1801 até 1890 considerou-se que a população cresceu a uma taxa de $2,2 \%$ ao ano, conforme consta nas estimativas provinciais para o período de 1818 a 1872 para o Rio Grande do Norte (RODARTE, 2008). Portanto, de posse da população do Rio Grande do Norte em 1872, redistribuída proporcionalmente por grupo etário conforme a distribuição etária de 1890, decrementou-se a população de cada faixa etária por ano, até 1805 , utilizando uma taxa de crescimento anual de $2,2 \%$. 0 próximo item apresenta a metodologia empregada para a correção dos óbitos.

\section{Correção dos dados de óbitos}

Para a análise da mortalidade foram utilizadas informações de óbitos contidas no mapa estatístico de 1805, por considerá-las mais coerentes do que aquelas referentes ao mapa estatístico de 1801, que apresentava poucos casos, sobretudo, para a população infantil. Inicialmente, para correção da mortalidade adulta (grupos etários acima de 20 anos), adotou-se o método de Growth Balance de Brass (1975), que tem como pressuposto o fato de que é possível ajustar a diferença de cobertura entre os óbitos e a população, possibilitando o cálculo da mortalidade específica por taxas corrigidas por idade, considerando que a população é estável e fechada. Sendo assim, a cobertura de óbitos não varia com a idade. A ideia principal é que, em uma população fechada ou com fluxo migratório pequeno, as formas de entrada da população são os nascimentos e as saídas correspondem aos óbitos.

Nesse sentido, a taxa de mudança da população em dois pontos do tempo é igual à diferença entre as taxas de entrada e as taxas de saída durante o intervalo considerado (BRASS, 1975). Dessa forma, o método baseia-se na equação geral da população:

$P_{2}=P_{1}+B_{1}-D$

Em que:

$P_{1}=$ população inicial;

$P_{2}=$ população final;

$B=$ número de nascimentos durante o período entre $P_{1}$ e $P_{2}$;

$D=$ número de óbitos durante o período entre $P_{1}$ e $P_{2}$.

Seguindo a lógica de que o número de nascimentos é igual ao número de óbitos de uma população em idade $x$, a taxa de entrada da população com $x$ anos e mais de idade é dada pelas pessoas com exatamente $x$ anos de idade completos e as saídas são os óbitos dos indivíduos com $x$ anos e mais: 


$$
\frac{N(x)}{N(x+)}=r(x+)+\frac{D(x+)}{N(x+)}
$$

\section{Em que:}

$N(x)=$ pessoas com exatamente $x$ anos de idade;

$N(x+)=$ número total de indivíduos acima da idade $x$;

$D(x+)=$ número de óbitos da população com idade de $x$ anos e mais;

$r=$ taxa de crescimento na idade de $x$ anos e mais.

Nesta perspectiva, percebe-se que as taxas de entrada e de saída estão linearmente relacionadas. No entanto, considerando-se a existência de sub-registros nos dados de mortalidade, a equação modifica-se para:

$D(x+)=C(\chi) \cdot D(x+)$

Em que:

$D(x+)=$ total de óbitos registrados;

$D(x+)=$ número total de óbitos a partir da idade $x$;

$C(x)=$ grau de cobertura de óbito para idade de $x$ anos e mais.

Como é assumido que o grau de cobertura $C(x)$ dos óbitos é igual em todas as idades, o $C(x)$ pode ser substituído por uma constante $C$, que não apresenta variação com a idade. Nesse caso, a fórmula modifica-se novamente para:

$\frac{N(x)}{N(\chi+)}=r+\frac{1}{C} \star \frac{D^{*}(\chi+)}{N(x+)}$

Em seguida, para estimar a mortalidade infanto-juvenil (abaixo de 20 anos), a estratégica metodológica foi utilizar uma função de mortalidade já existente, tendo como critério de escolha aquela que mais se aproximava da função de mortalidade estimada para a mortalidade adulta. Após a análise de diversos níveis das tábuas de vida de Coale e Demeny (1996) do modelo oeste (6) e leste (18), e de 19 funções de mortalidade de diferentes países disponíveis no site The Human Mortality Database, adotou-se a função da Islândia em 1860.

Por fim, para compatibilizar as duas partes da função de mortalidade (infanto-juvenil e adulta), utilizou-se técnica de padronização indireta, sob o pressuposto de que a estrutura da função de mortalidade escolhida para representar a mortalidade das idades abaixo de 20 anos também era adequada para representar a mortalidade das idades acima de 20 anos.

A padronização indireta parte do pressuposto de que, para estimar uma determinada função, é necessário adotar de outra população uma função conhecida de taxas específicas e supor que ambas as funções tenham a mesma estrutura (CARVALHO; SAWYER; RODRIGUES, 1998). Para ajustar o nível da função como um todo, foram utilizadas apenas as informações de mortalidade acima dos 20 anos, visto que para tais grupos etários têm-se os “óbitos observados corrigidos" e óbitos estimados com base na função adotada como padrão. 
Pressupõe-se que, para cada grupo de idade $x$, a função (taxas específicas) estimada da população em estudo será um múltiplo, segundo uma constante $K$ da função padrão tomada emprestada. Então, calculado o $K$, podem-se obter as taxas específicas de mortalidade por meio da seguinte equação:

${ }_{n} M_{x, \text { est }}=K \cdot{ }_{n} M_{x, S}$

Em que:

${ }_{n} M_{x, e s t}=$ função estimada para o grupo etário $(x ; x+n)$ da população em estudo;

${ }_{n} M_{x, S}=$ função padrão para o grupo etário $(x ; x+n)$ da população tomada como padrão;

$K=$ constante que representa a diferença de nível entre as funções das duas populações.

Para calcular $K$, é preciso primeiramente calcular o total de eventos esperados desde que o nível e o padrão da mortalidade da população em estudo forem iguais ao nível da mortalidade padrão (neste caso, $K=1$ ). 0 quociente entre os óbitos observados corrigidos acima de 20 anos e os óbitos esperados estimados para as idades acima de 20 anos será o valor de $K$.

$\mathrm{K}=\frac{O b_{o b s}}{\bar{O} b_{e s t}}$

Em que:

$O b_{o b s}=$ óbitos observados e corrigidos na população em estudo;

$\bar{O} b_{\text {est }}=$ óbitos esperados estimados.

Assim, obteve-se uma nova função de mortalidade para o estado do Rio Grande do Norte em 1805, que foi utilizada para estimar as expectativas de vida por grupo etário. Se a população do Rio Grande do Norte está sendo considerada quase-estável durante o período de 1801 a 1890, significa que a mortalidade também era constante e, por isso, a função de mortalidade estimada para 1805 deve representar o comportamento da mortalidade durante todo o período.

A próxima seção apresenta os principais resultados: estimativas de população; função de mortalidade estimada após a compatibilização das funções corrigidas de mortalidade adulta e infanto-juvenil de 1801 a 1890; além da tabela de vida para o Rio Grande do Norte nesse período, incluindo a esperança de vida ao nascer.

\section{Principais resultados}

\section{Análise descritiva}

O Gráfico 1 compara as pirâmides etárias da população do Rio Grande do Norte em 1801,1805 e $1872 .^{3}$ Nota-se, sobretudo para os dados provenientes dos mapas de 1801 e 1805, um comportamento mais oscilante da participação relativa dos grupos etários no

\footnotetext{
${ }^{3}$ Não foram incluídas informações sobre população do Rio Grande do Norte por idade e sexo para 1890 em função da indisponibilidade de dados.
} 
total da população, sinalizando uma possível incompletude das informações provenientes dessas fontes documentais. Por exemplo, de 1801 para 1805 a base da pirâmide alarga-se sobremaneira, o que possivelmente se deve mais a uma melhor cobertura da população menor de dez anos de idade no mapa de 1805 do que a uma possível maior sobrevivência dos indivíduos desse grupo etário ou aumento no número de nascimentos, no decurso de apenas cinco anos.

\section{GRÁFICO 1}

Pirâmide etária da população total, por sexo

Rio Grande do Norte - 1801-1872

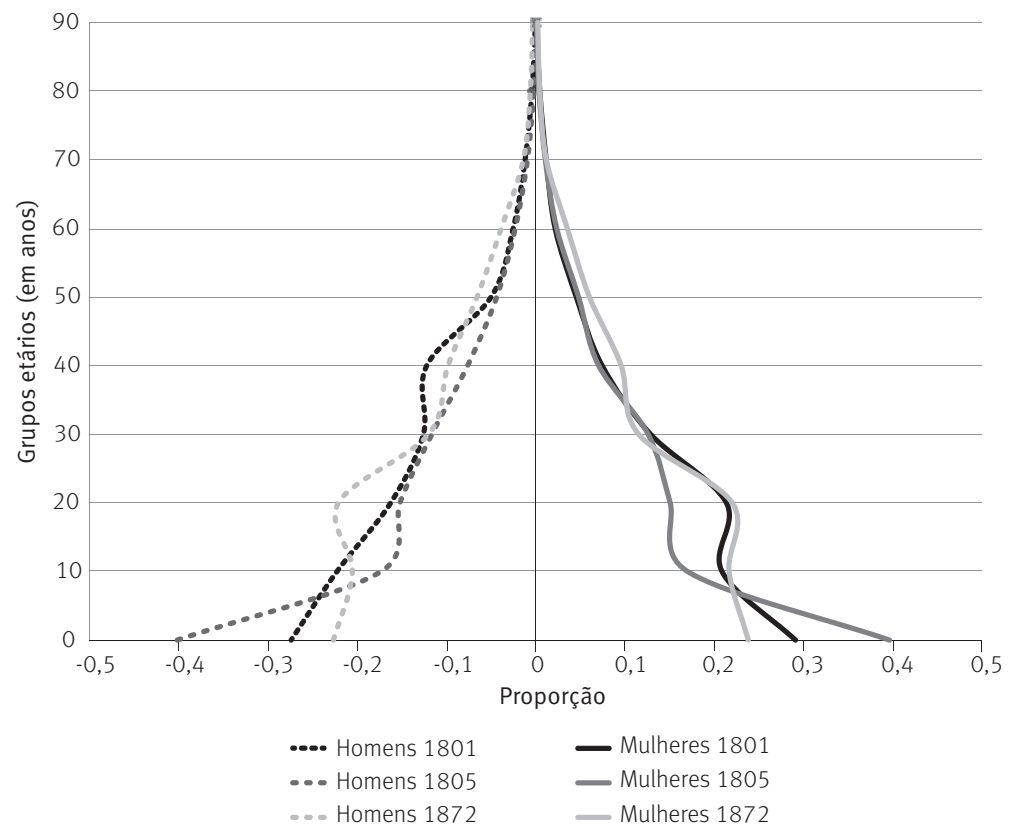

Fonte: 1801: AHU. ACL. CU. B. Rio Grande do Norte, Caixa 9, Documento 565; 1805: AHU. ACL. CU. B. Rio Grande do Norte, Caixa 10, Documento 629; 1872: Censo Imperial de 1872.

Quando comparada a estrutura etária da província norte-rio-grandense segundo o Censo Imperial de 1872 com a estrutura etária obtida pelos mapas de população, percebe-se uma base de pirâmide mais estreita segundo dados censitários e maior oscilação da população entre 10 e 30 anos de idade. Porém, obtendo-se a razão de sexo por grupos etários nos três pontos no tempo, o comportamento é mais estável para 1872 do que para 1801 e 1805 (Gráfico 2).

Nos dados de 1872, há em praticamente todos os grupos etários excesso de homens (valores de razão de sexo maiores do que a unidade), e com tendência de crescimento nas idades mais avançadas. Nos dados dos mapas de população, mesmo considerando grupos etários decenais, a tendência não é suave, sendo discrepantes, para o mapa de 1801, a baixa razão de sexo no grupo etário 30 a 49 anos $(0,74)$, indicando um excesso de mulheres nessas idades, e a elevada razão entre população masculina e feminina no grupo etário 50 
a 59 anos $(1,59)$ (Gráfico 2). Conforme anteriormente mencionado para a população menor de dez anos, esses indícios de subenumeração para grupos etários específicos no mapa de 1801 apontam para uma baixa qualidade das informações contidas nesse mapa em comparação com o de 1805, ao menos. Inclusive, conforme mostra a Tabela 1, a diferença entre a população observada nos dois mapas utilizados e a população estimada indica que justamente em 1801 o grau de cobertura foi de apenas $60 \%$, enquanto para o mapa de 1805 correspondeu a $92 \%$.

\section{GRÁFICO 2}

Razão de sexo da população total, por grupos etários Rio Grande do Norte - 1801-1872

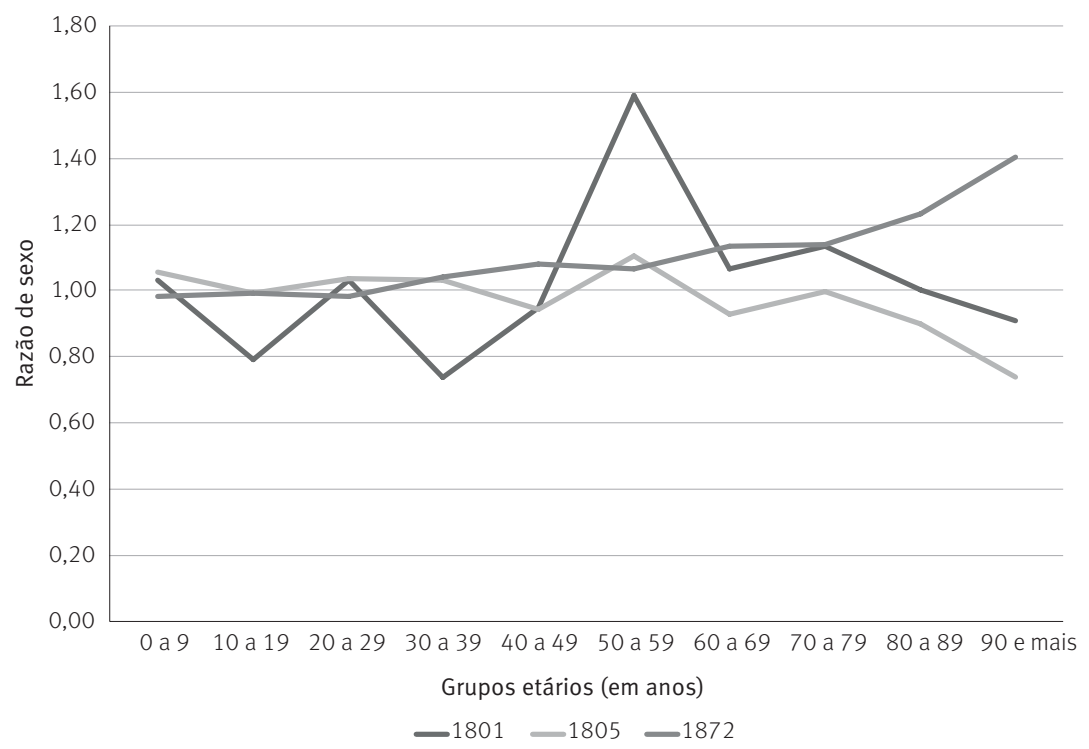

Fonte: 1801: AHU. ACL. CU. B. Rio Grande do Norte, Caixa 9, Documento 565; 1805: AHU. ACL. CU. B. Rio Grande do Norte, Caixa 10, Documento 629; 1872: Censo Imperial de 1872.

TABELA 1

População total observada e estimada Rio Grande do Norte - 1801-1805

\begin{tabular}{|c|c|c|}
\hline População & 1801 & 1805 \\
\hline Observada & 29.443 & 49.250 \\
\hline Estimada & 49.004 & 53.461 \\
\hline Diferença & 19.561 & 42.11 \\
\hline Grau de cobertura (\%) & 60,0 & 92,0 \\
\hline
\end{tabular}

Fonte: 1801: AHU. ACL. CU. B. Rio Grande do Norte, Caixa 9, Documento 565; 1805: AHU. ACL. CU. B. Rio Grande do Norte, Caixa 10, Documento 629; 1872: Censo Imperial de 1872; 1890: Censo de 1890 e Relatórios de Presidentes da Província do Rio Grande do Norte.

No que se refere às demais características da população do Rio Grande do Norte de acordo com os mapas estatísticos utilizados e o Censo Imperial de 1872, verifica-se uma proporção muito baixa de escravos: 17\% em 1801, 15\% em 1805 e 3\% em 1872. 
Possivelmente, a subenumeração desses indivíduos era maior no conjunto da população, sobretudo nos dados referentes ao início do século XIX, o que reforça ainda mais a hipótese de baixa qualidade das informações quanto ao grau de cobertura. 0 próximo item apresenta as estimativas de mortalidade

\section{Estimativas de mortalidade}

O Gráfico 3 mostra a função de mortalidade estimada que representa os níveis e padrões de mortalidade no Rio Grande do Norte entre 1801 e 1890, sob o pressuposto de que sua população era estável nesse período. De acordo com as taxas específicas de mortalidade (TEM) estimadas, a mortalidade no Rio Grande do Norte era mais intensa na população infantil e naquela a partir de 50 anos, idades mais suscetíveis às condições adversas como as secas e as doenças infecciosas que se fizeram presentes durante boa parte do período colonial e provincial. Verificam-se diferenças entre as TEM observadas e as estimadas em todos os grupos etários, principalmente de 0 a 4 anos e acima de 50 anos. Esses resultados sugerem que a intensidade da mortalidade no Rio Grande do Norte era maior do que aquela registrada no mapa de população e que o sub-registro era concentrado nas idades mais jovens e avançadas.

GRÁFICO 3

Taxas específicas de mortalidade estimadas e observadas Rio Grande do Norte - 1805-1890

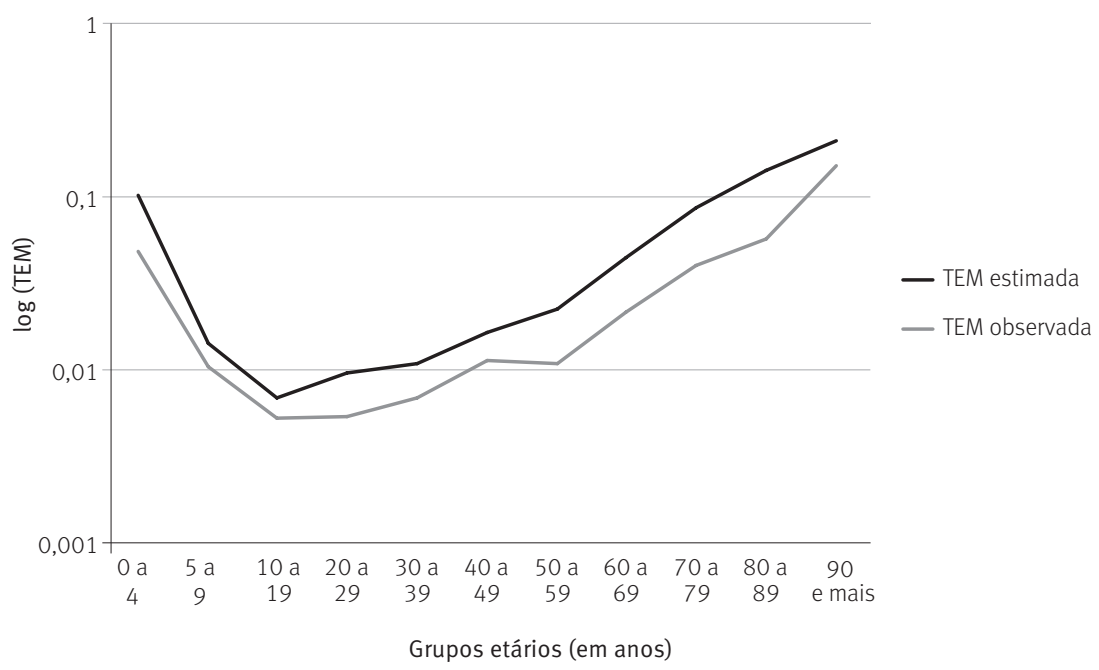

Fonte: 1801: AHU. ACL. CU. B. Rio Grande do Norte, Caixa 9, Documento 565; 1805: AHU. ACL. CU. B. Rio Grande do Norte, Caixa 10, Documento 629; 1872: Censo Imperial de 1872; 1890: Censo de 1890 e Relatórios de Presidentes da Província do Rio Grande do Norte.

Com a obtenção da nova função de mortalidade para o Rio Grande do Norte, foi possível estimar a expectativa de vida da população durante todo o período em estudo, que se insere na fase de pré-transição demográfica. De acordo com a Tabela 2, a expectativa de vida ao nascer estimada para o Rio Grande do Norte foi de 32,6 anos, um valor muito 
próximo das estimativas de Mortara (1941) para o Brasil entre 1870 e 1890 utilizando duas tábuas distintas de mortalidade: 32,7 e 33,9 anos.

TABELA 2

Tabela de sobrevivência

Rio Grande do Norte - 1801-1890

\begin{tabular}{cccccccccc}
\hline $\mathrm{x}$ & $\mathrm{n}$ & ${ }_{\mathrm{n}} \mathrm{m}_{\mathrm{x}}$ & $\mathrm{n}_{\mathrm{n}}$ & $\mathrm{n}_{\mathrm{n}} \mathrm{q}_{\mathrm{x}}$ & \multicolumn{1}{c}{$\mathrm{l}_{\mathrm{x}}$} & ${ }_{\mathrm{n}} \mathrm{d}_{\mathrm{x}}$ & ${ }_{\mathrm{n}} \mathrm{L}_{\mathrm{x}}$ & $\mathrm{T}_{\mathrm{x}}$ & $\mathrm{e}_{\mathrm{x}}$ \\
\hline 0 & 5 & 0,10272 & 2,5 & 0,4087 & 100000 & 40867 & 397832 & 3257507 & 32,58 \\
5 & 5 & 0,01412 & 2,5 & 0,0682 & 59133 & 4031 & 285587 & 2859675 & 48,36 \\
10 & 10 & 0,00691 & 5,0 & 0,0667 & 55102 & 3678 & 532628 & 2574088 & 46,72 \\
20 & 10 & 0,00972 & 5,0 & 0,0927 & 51424 & 4767 & 490405 & 2041460 & 39,70 \\
30 & 10 & 0,01091 & 5,0 & 0,1034 & 46657 & 4826 & 442443 & 1551055 & 33,24 \\
40 & 10 & 0,01644 & 5,0 & 0,1519 & 41832 & 6355 & 386543 & 1108612 & 26,50 \\
50 & 10 & 0,02259 & 5,0 & 0,2029 & 35477 & 7200 & 318773 & 722069 & 20,35 \\
60 & 10 & 0,04479 & 5,0 & 0,3659 & 28277 & 10347 & 231038 & 403297 & 14,26 \\
70 & 10 & 0,08685 & 5,0 & 0,6055 & 17930 & 10857 & 125014 & 172259 & 9,61 \\
80 & 10 & 0,14147 & 5,0 & 0,8286 & 7073 & 5860 & 41426 & 47245 & 6,68 \\
90 & $\infty$ & 0,20837 & 4,8 & 1,0000 & 1212 & 1212 & 5819 & 5819 & 4,80 \\
\hline
\end{tabular}

Fonte: 1801: AHU. ACL. CU. B. Rio Grande do Norte, Caixa 9, Documento 565; 1805: AHU. ACL. CU. B. Rio Grande do Norte, Caixa 10, Documento 629; 1872: Censo Imperial de 1872 e Relatórios de Presidentes da Província do Rio Grande do Norte.

Esse resultado é compatível com o esperado para um período de pré-transição demográfica, sendo que a semelhança entre os valores das esperanças de vida estimadas por esse estudo e aquela estimada por Mortara (1941), também para o século XIX, corrobora o argumento de que, no período anterior ao declínio consistente do volume de óbitos, a mortalidade oscilava pouco no tempo e, ao menos com base nesses achados, também entre regiões. Na comparação internacional, a esperança de vida ao nascer de cerca de 33 anos para o Rio Grande do Norte, de 1801 a 1870, também se mostra um resultado coerente. Ainda conforme apresentado por Mortara (1941), em países mais avançados durante o século XIX, como Inglaterra e França, a expectativa de vida era bem mais elevada (44,16 e 42,13 anos, respectivamente), enquanto em países da Europa oriental, menos desenvolvidos, os valores eram menores: 27,65 anos na Rússia e 27,65 anos na Áustria.

\section{Conclusão}

De um modo geral, o presente artigo alcançou o objetivo de estimar indicadores de mortalidade para o Rio Grande do Norte no contexto de pré-transição demográfica. A despeito da árdua a tarefa de lidar com informações cujas fontes não tenham sido desenhadas propriamente para fins de análise demográfica (no caso dos mapas de população) ou com recenseamentos delineados antes da era moderna das pesquisas censitárias, os resultados encontrados se mostraram coerentes.

Embora seja importante reconhecer as possíveis limitações desse trabalho, como, por exemplo, a ausência de informações de dados de outros componentes da dinâmica 
demográfica para o período que pudessem ter sido incorporados nas análises, como a fecundidade e a migração, os resultados obtidos contribuem para as reflexões quantitativas sobre o passado brasileiro. Sugere-se, portanto, que trabalhos futuros se dediquem ao levantamento de fontes de informações em que possam ser obtidos, para os períodos proto e pré-estatístico, dados acerca dos fluxos migratórios e das experiências de fecundidade, como, por exemplo, os registros paroquiais e novos mapas de população.

Outro ponto importante a se destacar diz respeito à metodologia utilizada. Por exemplo, para estimar a mortalidade há vários métodos, além do adotado no presente estudo, que podem ser empregados, tais como o de gerações extintas de Preston et al. (1980), Bennett e Houriuchi (1981), Hill (1987) e Hill e Choi (2004). E o teste de outros métodos pode ser uma demanda para trabalhos futuros com o intuito de verificar se os resultados encontrados continuam ou não consistentes. Inclusive, a esse respeito, cabe mencionar que, apesar do estudo de Mortara (1941), que antecede cronologicamente os métodos correntemente utilizados para a estimação da mortalidade como o método de Growth Balance de Brass (1975), os resultados encontrados pelo autor para o Brasil foram bastante semelhantes ao do Rio Grande do Norte em relação à esperança de vida ao nascer, o que inicialmente reforça a coerência do indicador encontrado.

Por fim, recomendam-se novos estudos sobre o período de pré-transição demográfica para outras localidades do país e para além das capitanias (e províncias) do centro-sul do Brasil. A demografia formal conta com inúmeras opções de técnicas para lidar com dados com problemas em sua qualidade de registro, e elas podem ser bastante úteis para compreender, do ponto de vista quantitativo, a dinâmica demográfica em tempos pretéritos como o de pré-transição.

\section{Referências}

ALDEN, D. The population of Brazil in the late eighteen century: a preliminary study. Hispanic American Historical Review, v. 43, p. 173-205, maio 1963.

ALVES, J. E. D. A polêmica Malthus versus Condorcet reavaliada à luz da transição demográfica. Rio de Janeiro: IBGE, Escola Nacional de Ciências Estatísticas, 2002 (Textos para discussão, n. 4).

ARAÚJO, A. I. C.; MACEDO, M. K. O sertão febril: impacto microbiano e escravidão nos espaços (in)salubres da Província do Rio Grande do Norte, Ribeira do Seridó (1856-1888). Mneme - Revista de Humanidades, v. 12, n. 30, p. 343-352, jul./dez. 2011.

BOTELHO, T. R. População e nação no Brasil do século XIX. 1998. 241 f. Tese (Doutorado em História) - Departamento de Ciências Sociais da Faculdade de Filosofia, Letras e Ciências Humanas, Universidade de São Paulo, São Paulo, 1998.

BRASS, W. Methods for estimating fertility and mortality from limited and defective data. Chapell Hill: University of North Carolina at Chapell Hill, International Program of Laboratories for Population Statistics, 1975.

CARVALHO, J. A. M; SAWYER, D. O.; RODRIGUES, R. N. Introdução a alguns conceitos básicos e medidas em demografia. Belo Horizonte: Abep, 1998 (Série Textos Didáticos, n. 1). 
CASCUDO, L. C. Capítulo XI. História do Rio Grande do Norte. 2. ed. Natal/Rio de Janeiro: Fundação José Augusto; Achiame, 1984. p. 275-289.

COALE, A. J.; DEMENY, P. Regional model life tables and stable population. New Jersey: Princeton University Press, 1966.

DIAS, P. de O. Onde fica o sertão rompem-se as águas: processo de territorialização da ribeira do Apodi-Mossoró (1676-1725). Dissertação (Mestrado) - Programa de Pós-graduação em História - PPGH, Universidade Federal do Rio Grande do Norte, Natal, 2015.

DIAS, T. A. Dinâmicas mercantis coloniais: capitania do Rio Grande do Norte (1760-1821). Dissertação (Mestrado) - Programa de Pós-graduação em História - PPGH, Universidade Federal do Rio Grande do Norte, Natal, 2011.

FRANCO, O. História da febre amarela no Brasil. Revista Brasileira de Malariologia e Doenças Tropicais, 1969.

GUERRA, F. Secas do Nordeste. In: ROSADO, V. (Org.). Memorial da seca. Brasília: Centro Gráfico do Senado Federal/ESAM, 1981 (Coleção Mossoroense).

HILL, K.; CHOI, Y. Death distribution methods for estimating adult mortality: sensitivity analysis with simulated data errors. Adult mortality in developing countries workshop. Marin County, California: The Marconi Center, July 2004.

HILL, K. Estimating census and death registration completeness. Asian and Pacific Population Forum, v. 1, n. 3, p. 8-12, 1987.

LYRA, A.T. de. A independência do Brasil no Rio Grande do Norte. Rio de Janeiro: Pongetti, 2011. . As secas do Nordeste. In: ROSADO, V. (Org.). Memorial da seca. Brasília: Centro Gráfico do Senado Federal/ESAM, 1981 (Coleção Mossoroense).

História do Rio Grande do Norte. 3. ed. Natal: EDUFRN, 2008.

MACEDO, H. A. M. de. História indígena no sertão da capitania do Rio Grande após as guerras dos bárbaros. In: MACEDO, H. A. M. de; ARAUJO, M. A. A.; SANTOS, R. da S. (Org.). Seridó potiguar: tempos, espaços, movimentos. 1. ed. João Pessoa: Ideia Editora, 2011. v. 1.

MARCÍLIO, M. L. Crescimento histórico da população brasileira até 1872. Cadernos Cebrap, n. 16, 1973.

MEDEIROS, T. Aspectos geopolíticos e antropológicos da história do Rio Grande do Norte. Natal: Imprensa Universitária, 1973.

MONTEIRO, D. M. Introdução à história do Rio Grande do Norte. 2. ed. Natal: Coopertativa Cultural, 2002.

MORTARA, G. Estudos sobre a utilização do censo demográfico para a reconstrução das estatísticas do movimento da população do Brasil. VII Tabelas de mortalidade e de sobrevivência para os períodos de 1870-1890 e 1890-1920. Cálculo, exame e comparações internacionais. Revista Brasileira de Estatística, ano II, n. 7, p. 494-538, 1941.

NADALIN, S. O. História e demografia: elementos para um diálogo. Campinas: Associação Brasileira de Estudos Populacionais - Abep, 2004.

PAIVA, C. A. et al. Publicação crítica do recenseamento geral do Império do Brasil de 1872. Belo Horizonte: Cedeplar/UFMG, 2012.

PAIVA, Y. A demografia da Paraíba. Uma análise preliminar, 1774-1820. Anais de Historia de Alem-Mar, n. 16, p. 205-226, 2015. 
PRATA, P. A transição epidemiológica no Brasil. Cadernos de Saúde Pública, v. 8, n. 2, p. 168-175, 1992.

PRESTON, S. et al. Estimating the completeness of reporting of adult deaths in populations that are approximately stable. Population Studies, v. 46, n. 2, p. 179-202, 1980.

RODARTE, M. M. S. 0 trabalho do fogo: perfis de domicílios enquanto unidades de produção e reprodução na Minas Gerais Oitocentista. Tese (Doutorado) - Programa de Pós-graduação em Demografia -PPGD, Universidade Federal de Minas Gerais - UFMG, Belo Horizonte, 2008.

SANTOS, A. R. Cemitérios no Seridó, século XIX: construindo de uma pesquisa. Revista Inter-Legere, p. 48-76, jan./jun. 2013.

SANTOS, P. P. dos. Evolução econômica do Rio Grande do Norte. Natal: Clima, 1994.

WOOD, C. H.; CARVALHO, J. A. M. A demografia da desigualdade no Brasil. Rio de Janeiro: Ipea, 1994.

\section{Sobre as autoras}

Luciana Conceição de Lima é doutora e mestre em Demografia pela Universidade Federal de Minas Gerais (UFMG). Professora adjunta do Departamento de Demografia e Ciências Atuariais da Universidade Federal do Rio Grande do Norte (UFRN) e membro permanente do Programa de Pós-Graduação em Demografia (PPGDEM) da UFRN.

Dayane Julia Carvalho Dias é doutoranda em Demografia no Núcleo de Estudos de População "Elza Berquó" (Nepo), da Universidade Estadual de Campinas (Unicamp) e mestre em Demografia pelo Programa de Pós-Graduação em Demografia da Universidade Federal do Rio Grande do Norte (UFRN).

Luana Junqueira Dias Myrrha é doutora e mestre em Demografia pela Universidade Federal de Minas Gerais (Cedeplar/UFMG). Professora adjunta I no Departamento de Demografia e Ciências Atuariais da Universidade Federal do Rio Grande do Norte (UFRN), coordenadora da graduação em Ciências Atuariais da UFRN e membro permanente do Programa de Pós-Graduação em Demografia (PPGDEM) da UFRN.

\section{Endereço para correspondência}

Luciana Conceição de Lima

Centro de Ciências Exatas e da Terra, Departamento de Demografia e Ciências Atuariais Avenida Senador Salgado Filho, 3000, Candelária

59066-800 - Natal-RN, Brasil

Dayane Julia Carvalho Dias

Unicamp - Universidade Estadual de Campinas, Núcleo de Estudos de População “Elza Berquó"

Av. Albert Einstein, 1300, Cidade Universitária Zeferino Vaz

13081-970, Cx. Postal 6166 - Campinas-SP, Brasil

Luana Junqueira Dias Myrrha

Centro de Ciências Exatas e da Terra, Departamento de Demografia e Ciências Atuariais Avenida Senador Salgado Filho, 3000, Candelária

59066-800 - Natal-RN, Brasil 


\begin{abstract}
Mortality estimations for Rio Grande do Norte in a context of demographic pre-transition

For periods prior to the beginning of the demographic transition, there are few quantitative studies about the impact of mortality on the population. Based on this absence, this article estimates mortality indicators for Rio Grande do Norte in the context of demographic pre-transition, using nineteenth-century data from statistical population maps (1801 and 1805) and census (1872 and 1890). In order to deal with population and death data, Brass's Growth Balance method (1975) was used, as well as Coale and Demeny's life tables (1996), functions extracted from The Human Mortality Database and indirect standardization technique. As a result, it was verified that mortality in Rio Grande do Norte was more intense in child and elderly population, over 50 years old, which represent the most vulnerable populations to adverse conditions such as droughts and infectious diseases. Life expectancy at birth is observed at 32.6 years old, a value very close to Mortara's (1941) estimates for Brazil between 1870 and 1890. Further studies are suggested for the demographic and major pre-transition period as well as the use of demographic techniques in studies focused on the Brazilian past.
\end{abstract}

Keyword: Demographic pre-transition. Mortality. Rio Grande do Norte. Demographic techniques.

\title{
Resumen
}

Estimaciones de mortalidad para Rio Grande do Norte en un contexto previo a la transición demográfica

Para los períodos anteriores al comienzo de la transición demográfica hay pocos estudios cuantitativos sobre el impacto de la mortalidad en la población. Sobre la base de esta brecha, este artículo estimó indicadores de mortalidad para Rio Grande do Norte, en el contexto previo a la transición demográfica, a partir de datos del siglo XIX: informes estadísticos de población (1801 y 1805) y censos (1872 y 1890). Para hacer frente a la información sobre población y muerte se utilizaron el método de Growth Balance de Brass (1975) y las tablas de vida de Coale Demeny (1996), se extrajeron funciones de The Human Mortality Database y técnicas de estandarización indirecta. Como resultado, se verificó que la mortalidad en Rio Grande do Norte fue más intensa en niños y personas mayores de 50 años, que representan a los más vulnerables frente las condiciones adversas como la sequía y las enfermedades infecciosas. También se obtuvo una esperanza de vida al nacer de 32,6 años, valor muy próximo a las estimaciones de Mortara (1941) para Brasil entre 1870 y 1890 . Se sugieren más estudios para la pretransición demográfica y mayor utilización de técnicas cuantitativas en los estudios del pasado brasileño.

Palabras clave: Pretransición demográfica. Mortalidad. Rio Grande do Norte. Técnicas demográficas. 


\section{Anexo}

TABELA 1

Aplicação do Método de Growth Balance de Brass (1975)

Rio Grande do Norte - 1805

\begin{tabular}{|c|c|c|c|c|c|c|c|c|c|c|c|}
\hline \multirow[b]{2}{*}{ Faixa etária } & \multicolumn{2}{|c|}{$\begin{array}{l}\text { População } \\
\text { observada }\end{array}$} & \multicolumn{2}{|c|}{$\begin{array}{l}\text { População } \\
\text { acumulada }\end{array}$} & \multirow{2}{*}{$\begin{array}{c}\text { Taxa de } \\
\text { crescimento } \\
\text { r }\end{array}$} & \multirow{2}{*}{$\begin{array}{c}\begin{array}{c}\text { Óbito } \\
\text { observado }\end{array} \\
5 \mathrm{Dx} \\
1805 \\
\end{array}$} & \multirow{2}{*}{$\begin{array}{c}\begin{array}{c}\text { Óbito } \\
\text { acumulado }\end{array} \\
\text { wDx } \\
1805 \\
\end{array}$} & \multirow[b]{2}{*}{$\mathbf{x}$} & \multirow[b]{2}{*}{$\begin{array}{c}n(X) \\
1805\end{array}$} & \multirow{2}{*}{$\begin{array}{c}Y \\
N(x) / \\
N(X+)-r\end{array}$} & \multirow{2}{*}{$\begin{array}{c}\mathrm{X} \\
\mathrm{D}(\mathrm{x}+) / \\
\mathrm{N}(\mathrm{x}+)\end{array}$} \\
\hline & $\begin{array}{l}N(x) \\
1801\end{array}$ & $\begin{array}{l}N(x) \\
1805\end{array}$ & $\begin{array}{l}w N x \\
1801\end{array}$ & $\begin{array}{l}\text { wNx } \\
1805\end{array}$ & & & & & & & \\
\hline 0 a 4 anos & 7.820 & 8.531 & 49.004 & 53.461 & 0,022 & 410 & 804 & 0 & 4.351 & 0,05938 & 0,01503 \\
\hline 5 a 09 anos & 8.132 & 8.871 & 41.185 & 44.930 & 0,022 & 93 & 394 & 5 & 4.878 & 0,08657 & 0,00876 \\
\hline 10 a 19 anos & 9.755 & 10.642 & 33.053 & 36.059 & 0,022 & 56 & 301 & 10 & 5.190 & 0,12193 & 0,00834 \\
\hline 20 a 29 anos & 9.275 & 10.118 & 23.298 & 25.417 & 0,022 & 54 & 245 & 20 & 4.153 & 0,14141 & 0,00962 \\
\hline 30 a 39 anos & 5.954 & 6.495 & 14.024 & 15.299 & 0,022 & 45 & 191 & 30 & 2.653 & 0,15138 & 0,01246 \\
\hline 40 a 49 anos & 3.772 & 4.115 & 8.070 & 8.804 & 0,022 & 47 & 146 & 40 & 1.655 & 0,16595 & 0,01653 \\
\hline 50 a 59 anos & 2.295 & 2.504 & 4.298 & 4.689 & 0,022 & 27 & 99 & 50 & 970 & 0,18492 & 0,02102 \\
\hline 60 a 69 anos & 1.262 & 1.377 & 2.003 & 2.185 & 0,022 & 30 & 72 & 60 & 482 & 0,19845 & 0,03275 \\
\hline 70 a 79 anos & 504 & 550 & 741 & 808 & 0,022 & 22 & 42 & 70 & 189 & 0,21178 & 0,05144 \\
\hline 80 a 89 anos & 189 & 206 & 237 & 258 & 0,022 & 12 & 20 & 80 & 65 & 0,22800 & 0,07576 \\
\hline \multirow[t]{3}{*}{$\begin{array}{l}90 \text { anos e } \\
\text { mais }\end{array}$} & 48 & 52 & 48 & 52 & 0,022 & 8 & 8 & & & & \\
\hline & & & & & Média r & & & & & $\begin{array}{l}\text { Fator de } \\
\text { correção }\end{array}$ & $\begin{array}{c}\text { Grau de } \\
\text { cobertura }\end{array}$ \\
\hline & & & & & 0,022 & & & & & 0,5523 & 1,8107 \\
\hline
\end{tabular}

Fonte: AHU. ACL. CU. B. Rio Grande do Norte, Caixa 10, Documento 629. 
